\title{
Emerging Role of Laparoscopic and Robotic Surgery for Rectal Cancers
}

\author{
Glen C. Balch, MD \\ Department of Surgery, North Shore University Hospital, Northwestern University Feinberg School of Medicine, \\ Evanston, IL
}

The surgical management of rectal cancers is in a rapid phase of evolution that will increasingly deploy minimally invasive surgical (MIS) techniques. In this issue of Annals of Surgical Oncology are two articles about MIS approaches for rectal cancer, one comparing open versus laparoscopic proctectomy and the other comparing laparoscopic versus robotic approaches. ${ }^{1,2}$ These articles offer an opportunity to encourage the systematic validation of these technologies through clinical trials as they are increasingly adopted for colorectal surgery.

Over the past decade, urologists have made great strides in implementing robotic surgery for pelvic procedures, although the surgical oncology and colorectal surgery communities have not yet embraced this technology at the same pace. For example, in 2007, approximately 50,000 radical prostatectomies were performed with the Da Vinci robot system in the United States, or approximately $60 \%$ of radical prostatectomies. ${ }^{3}$ In general, the reported results of robotic prostatectomy demonstrated less blood loss and shorter length of hospital stay; although the potency and continence rates were comparable, a trend was noted toward a faster return of functional outcomes. ${ }^{3,4}$ The gynecological community has also started to use the robotic system, which has shown similar results to those described by urologists for prostatectomy. That is, compared with open or laparoscopic techniques, robotic surgery for radical hysterectomy resulted in less blood loss, fewer postoperative complications, and shorter length of stay. ${ }^{5-8}$

Last year, Mattias Soop and Heidi Nelson, in an editorial published in Annals of Surgical Oncology, wrote, "we

(C) Society of Surgical Oncology 2009

Published Online: 9 April 2009

G. C. Balch, MD

e-mail: GBalch@northshore.org believe that the cumulative literature now supports an equipoise on short term advantages and long-term oncologic outcomes (for laparoscopic proctectomy for cancer), making the case for large prospective randomized trials." ${ }^{9}$ Since the COST study group published its results in 2004 showing equivalence in oncologic outcomes between open and laparoscopic resection of colon cancer, the use of laparoscopic techniques for colon cancer has become popular and has now expanded to include rectal cancer patients. ${ }^{10-13}$ The data for rectal cancer is still being generated, but the randomized Conventional Versus Laparoscopic-Assisted Surgery in Colorectal Cancer (CLASICC trial), as well as several retrospective studies, demonstrate that the use of laparoscopic techniques has at least equivalent oncologic outcomes compared with an open laparotomy approach. ${ }^{12,14-16}$ For example, in this issue of Annals of Surgical Oncology is a multivariate analysis of 421 consecutive patients with stage II and stage III rectal cancer conducted by Law and colleagues who retrospectively compared outcomes of open versus laparoscopic resection. ${ }^{1}$ Laparoscopic resection was associated with significantly less blood loss and shorter hospital stay and was one of the independent significant factors associated with better survival $(P=.03$, hazard ratio $.558,95 \%$ confidence interval .339-.969). $\mathrm{Ng}$ and colleagues from Hong Kong reported last year in Annals of Surgical Oncology the preliminary results of a randomized trial comparing open versus laparoscopic abdominoperineal resection in 99 rectal cancer patients with a follow-up of 90 months. $^{12}$ Their results showed that postoperative recovery was better after laparoscopic surgery, and 5-year survival was identical. These results are consistent with other studies showing that laparoscopic colon resection provides better perioperative outcomes compared with open laparotomy, including decreased postoperative pain, earlier return to normal physical function, quicker return to bowel function, and shorter hospital stays. ${ }^{10-16}$ 
Laparoscopic total mesorectal excision is more technically difficult, and the learning curve is steep. Conventional laparoscopic surgery is limited in a confined surgical field as a result of fixed instrument tips, limited dexterity of the instruments, and a two-dimensional view. In contrast, the Da Vinci Surgical System (Intuitive Surgical, Sunnyvale, CA) overcomes these constraints because the instruments provide multiple degrees of freedom, three-dimensional imaging, ambidextrous capability, motion scaling, and tremor elimination. It may also provide a shorter learning curve if the start-up experience in robot-assisted radical prostatectomy is applicable in proctectomy. Although experienced laparoscopic surgeons may be able to overcome some of the limitations of the use of conventional laparoscopy in pelvis, the advantages of the robotic instrumentation may enable a larger number of surgeons to safely use this technology because the learning curve is less steep and shorter. It remains to be seen whether the robotassisted technique may be better than the conventional laparoscopic technique. However, it is most likely equivalent to rectal surgery of average complexity, and it provides yet another minimally invasive tool that may be preferable in more complicated rectal surgery, such as low rectal cancers.

In this issue, Baik and colleagues from Severance Hospital in Seoul, Korea, report their results on 113 patients with rectal cancer who were assigned to receive either robot-assisted low anterior resection (R-LAR, $n=56$ ) or laparoscopic low anterior resection (L-LAR, $n=57){ }^{2,17}$ They concluded that R-LAR was performed safely and effectively, and that perioperative outcomes with the robotic approach were comparable to L-LAR. ${ }^{2}$ Several other centers have described their early experience with robotic surgery in colorectal cancer and have concluded that it was both feasible and safe. ${ }^{17-20}$

The technological advantage telerobotic surgery provides is the ability to perform a fine dissection in a narrow surgical field. This theoretically translates into fewer conversions to an open procedure, fewer complications, and improved oncologic outcomes. In this comparative study by Baik and colleagues, some of these theoretical advantages seem to be fulfilled. As for perioperative outcomes, there was a significant difference in the serious complication rate and open conversion rate, both favoring the robotic approach. The serious complication rate in the laparoscopic group was $19.3 \%$ vs. $5.4 \%$ for the robotic group. The open conversion rate was $10.5 \%$ in the L-LAR group compared with $0 \%$ open conversion rate in the robotic group. The authors make a convincing case that the conversion to open laparotomy of most patients in the laparoscopic group was due to the patient's narrow pelvis, and that this conversion might have been avoided with the robot-assisted approach. Theoretically, autonomic nerve preservation will also be improved, but this was not reported in this study. There was no difference in the circumferential resection margin between the two groups. However, the robotic group had a significantly better macroscopic grading of the total mesorectal excision specimen. Again, we may infer that these factors play a role in long-term survival and local recurrence rates. Of course, longer follow-up and larger patient populations are needed for further evaluation.

Like many surgeons, I was initially skeptical whether robot-assisted surgery would provide much advantage over the conventional laparoscopic technique in complicated rectal surgery. However, as I continue to use the robot for low anterior resections, I have found that the system is indeed impressive, especially when operating in the narrow pelvis resecting a low rectal cancer. The many degrees of freedom permitted by the instruments make the operation easier. The visualization provided by a three-dimensional imaging system on a fixed camera platform is better than conventional laparoscopy. For the first time, I could perform a low anterior resection without suffering with low back or neck pain. This issue cannot be underestimated, as many surgeons who work in the pelvis routinely have lower back problems that may shorten their career. ${ }^{21}$ In the December 2008 issue of the American College of Surgeons' Surgery News, Adrian Park reports that "nearly 9 out of 10 laparoscopic surgeons said they experienced physical discomfort or symptoms related to performing surgery" according to the results of a survey of 317 laparoscopic surgeons.

This enthusiasm should not be interpreted as an unfettered acceptance of this new technology, for we are obligated to provide scientific evidence of its benefits through prospective clinical trials. The downsides of the robotic system are mainly cost, longer operating times, and loss of haptic feedback compared with conventional laparoscopic procedures. This is a sophisticated and expensive technology that requires training and supervision. There is a definite learning curve, which has been variously reported in the urologic and gynecologic literature at 25 to 50 cases. ${ }^{22}$ With experience, the operative times will decrease. In the Baik study, the mean operative time was virtually the same in the two groups $(190.1 \pm 45.0 \mathrm{~min}$ in the R-LAR group vs. $191.1 \pm 65.3 \mathrm{~min}$ in the L-LAR group; $P=.924)$. Last, the robotic instruments continue to evolve, and research is being performed to provide haptic feedback.

The leadership of the Society of American Gastrointestinal and Endoscopic Surgeons (SAGES) and the Minimally Invasive Robotic Association (MIRA) has written a thoughtful consensus statement about the use of robotic surgery, including recommendations for training, credentialing, and clinical applications. They stated that 
robotic surgery can serve "as an enabling technology for many surgeons, allowing them to provide complex minimally invasive procedures to a broad range of patients. The potential advantages of robotic surgery extend across many different surgical subspecialties." 23 Although this is a sophisticated technology with proven effectiveness in some areas of surgery, we are obligated to continue conducting prospective clinical trials involving colorectal surgery patients to verify that the benefits of this approach are clearly better than that of open and laparoscopic-assisted rectal surgery, and to learn the boundaries of patient selection and safety with this technique. All of us who use either robot-assisted or laparoscopic-assisted proctectomies should participate in the ACOSOG Z6051 or a similar trial.

\section{REFERENCES}

1. Law WL, Poon JTC, Fan JKM, et al. Comparison of outcome of open and laparoscopic resection for stage II and stage III rectal cancer. Ann Surg Oncol. 2009. DOI: 10.1245/s10434-009-0418-4.

2. Baik SH, Kwon HY, Kim JS, et al. Robotic versus laparoscopic low anterior resection of rectal cancer: short-term outcome of a prospective comparative study. Ann Surg Oncol. DOI:10.1245/ s10434-009-0435-3.

3. Wexner SD, Bergamaschi R, Lacy A, et al. The current status of robotic pelvic surgery: results of a multinational interdisciplinary consensus conference. Surg Endosc. 2009;23:438-43.

4. Hakimi AA, Blitstein J, Feder M, et al. Direct comparison of surgical and functional outcomes of robotic-assisted versus pure laparoscopic radical prostatectomy: single-surgeon experience. Urology. 2009;73:119-23.

5. Ko EM, Muto MG, Berkowitz RS, et al. Robotic versus open radical hysterectomy: a comparative study at a single institution. Gynecol Oncol. 2008;111:425-30.

6. Boggess JF, Gehrig PA, Cantrell L, et al. A comparative study of 3 surgical methods for hysterectomy with staging for endometrial cancer: robotic assistance, laparoscopy, laparotomy. Am J Obstet Gynecol. 2008;199:360.e1-360.e9.

7. Boggess JF, Gehrig PA, Cantrell L, et al. A case-control study of robot-assisted type III radical hysterectomy with pelvic lymph node dissection compared with open radical hysterectomy. Am J Obstet Gynecol. 2008;199:357.e1-7.
8. Visco AG, Advincula AP. Robotic gynecologic surgery. Obstet Gynecol. 2008;112:1369-84.

9. Soop M, Nelson H. Laparoscopic-assisted proctectomy for rectal cancer: on trial. Ann Surg Oncol. 2008;15:2357-9.

10. Fleshman J, Sargent DJ, Green E, et al. Laparoscopic colectomy for cancer is not inferior to open surgery based on 5-year data from the COST Study Group trial. Ann Surg. 2007;246:655-62.

11. Aziz O, Constantinides V, Tekkis PP, et al. Laparoscopic versus open surgery for rectal cancer: a meta-analysis. Ann Surg Oncol. 2006;13:413-24.

12. $\mathrm{Ng}$ SS, Leung KL, Lee JF, et al. Laparoscopic-assisted versus open abdominoperineal resection for low rectal cancer: a prospective randomized trial. Ann Surg Oncol. 2008;15:2418-25.

13. Nelson H, Sargent DJ, Wieand S, et al. A comparison of laparoscopically assisted and open colectomy for colon cancer. $N$ Engl J Med. 2004;350:2050-9.

14. Bonjer HJ, Hop WC, Nelson H, et al. Laparoscopically assisted vs open colectomy for colon cancer: a meta-analysis. Arch Surg. 2007;142:298-303.

15. Boller AM, Nelson H. Colon and rectal cancer: laparoscopic or open? Clin Cancer Res. 2007;13:6894s-6s.

16. Jayne DG, Guillou PJ, Thorpe $\mathrm{H}$, et al. Randomized trial of laparoscopic-assisted resection of colorectal carcinoma: 3-year results of the UK MRC CLASICC Trial Group. J Clin Oncol. 2007;25:3061-8.

17. Baik SH, Ko YT, Kang CM, et al. Robotic tumor-specific mesorectal excision of rectal cancer: short-term outcome of a pilot randomized trial. Surg Endosc. 2008;22:1601-8.

18. Spinoglio G, Summa M, Priora F, et al. Robotic colorectal surgery: first 50 cases experience. Dis Colon Rectum. 2008;51: 1627-32.

19. Hellan M, Anderson C, Ellenhorn JD, et al. Short-term outcomes after robotic-assisted total mesorectal excision for rectal cancer. Ann Surg Oncol. 2007;14:3168-73.

20. D'Annibale A, Morpurgo E, Fiscon V, et al. Robotic and laparoscopic surgery for treatment of colorectal diseases. Dis Colon Rectum. 2004;47:2162-8.

21. Berguer R, Smith W. An ergonomic comparison of robotic and laparoscopic technique: the influence of surgeon experience and task complexity. J Surg Res. 2006;134:87-92.

22. Lenihan JP Jr, Kovanda C, Seshadri-Kreaden U. What is the learning curve for robotic assisted gynecologic surgery? J Minim Invasive Gynecol. 2008;15:589-94.

23. Herron DM, Marohn M, SAGES-MIRA Robotic Surgery Consensus Group. A consensus document on robotic surgery. Surg Endosc. 2008;22:313-25. 